LAWRENCE LIVERMORE NAT IO N A L LABORATORY
Multi-fluid code simulations including anomalous non-diffusive transport of plasma and impurities in the tokamak SOL

A. Yu Pigarov, S.I. Krasheninnikov, T.D. Rognlien, W.P. West, B. LaBombard, B. Lipschultz, R. Maingi, V. Soukhanovskii

November 25, 2003

Contribution to Plasma Physics 
This document was prepared as an account of work sponsored by an agency of the United States Government. Neither the United States Government nor the University of California nor any of their employees, makes any warranty, express or implied, or assumes any legal liability or responsibility for the accuracy, completeness, or usefulness of any information, apparatus, product, or process disclosed, or represents that its use would not infringe privately owned rights. Reference herein to any specific commercial product, process, or service by trade name, trademark, manufacturer, or otherwise, does not necessarily constitute or imply its endorsement, recommendation, or favoring by the United States Government or the University of California. The views and opinions of authors expressed herein do not necessarily state or reflect those of the United States Government or the University of California, and shall not be used for advertising or product endorsement purposes. 


\title{
Multi-fluid code simulations including anomalous non-diffusive transport of plasma and impurities in the tokamak SOL
}

\author{
A. Yu. Pigarov ${ }^{* 1,2}$, S. I. Krasheninnikov ${ }^{1,2}$, T. D. Rognlien ${ }^{3}$, W. P. West ${ }^{4}$, B. LaBombard ${ }^{5}$, B. \\ Lipschultz $^{5}$, R. Maingi ${ }^{6}$, and V. Soukhanovskii ${ }^{7}$ \\ ${ }^{1}$ MAE Center for Energy Research, University of California at San Diego, EB-Unit-II, 9500 Gilman Dr., Mail \\ Stop 0417, La Jolla, CA 92093-0417, USA \\ 2 also at National Research Center "Kurchatov Institute", Moscow, Russia \\ ${ }^{3}$ Lawrence Livermore National Laboratory, Livermore, CA, USA \\ ${ }^{4}$ General Atomics, San Diego, CA, USA \\ ${ }^{5}$ Plasma Science and Fusion Center, Massachusetts Institute of Technology, Cambridge, MA, USA \\ ${ }^{6}$ Oak Ridge National Laboratory, Oak Ridge, TN, USA \\ ${ }^{7}$ Princeton Plasma Physics Laboratory, Princeton University, Princeton, NJ, USA
}

\begin{abstract}
Key words tokamak, edge plasma, cross-field transport, impurity, anomalous convection, fluid modeling PACS 52.55.Fa, 52.65.Kj
\end{abstract}

\begin{abstract}
Fast intermittent transport has been observed in the scrape-off layer (SOL) of major tokamaks including Alcator C-Mod, DIII-D, and NSTX. This kind of transport is not diffusive but rather convective. It strongly increases plasma flux to the chamber walls and enhances the recycling of neutral particles in the main chamber. We discuss anomalous cross-field convection (ACFC) model for impurity and main plasma ions and its relation to intermittent transport events, i.e. plasma density blobs and holes in the SOL. Along with plasma diffusivity coefficients, our transport model introduces time-independent anomalous cross-field convective velocity. In the discharge modelling, diffusivity coefficients and ACFC velocity profiles are adjusted to match a set of representative experimental data. We use this model in the edge plasma physics code UEDGE to simulate the multi-fluid two-dimensional transport for these three tokamaks. We present simulation results suggesting the dominance of anomalous convection in the far SOL transport. These results are consistent with the hypothesis that the chamber wall is an important source of impurities and that different impurity charge states have different directions of anomalous convective velocity.
\end{abstract}

\section{Introduction}

Fast intermittent cross-field plasma transport has been observed in the edge and scrape-off layer (SOL) of many tokamaks including Alcator C-Mod, DIII-D, and NSTX (see, respectively, the references [1], [2], and [3]) for details). The coherent plasma structures, called blobs [4], have been found to rapidly propagate (even ballistically) toward the wall. This kind of transport is convective rather than diffusive. It is characterized by strongly increased plasma fluxes to chamber walls and by enhanced recycling of neutrals in the main chamber $[1,5]$. The non-diffusive transport may significantly alter not only the particle recycling but also the degree of divertor detachment, plasma radiation loss, impurity sources and transport, peak power loads, and plasma-wall interactions.

The effects of non-diffusive intermittent transport on the macroscopic plasma transport and particle recycling occurring in the tokamak edge have been studied in Refs. [5, 6] with 2D fluid code UEDGE [8]. Along with anomalous plasma diffusivity coefficients $\left(D_{\perp}, \chi_{\perp}\right)$, our transport model introduces a time-independent anomalous cross-field convective (ACFC) velocity $V_{\text {conv }}$ [5]. For the main plasma ion specie, $V_{\text {conv }}$ is directed outwards. In discharge modeling, 2D profiles of plasma diffusivity coefficients and $V_{\text {conv }}$ are adjusted to match representative set of experimental data. Inclusion of $V_{\text {conv }}$ allowed us to reproduce properly not only individual properties of selected low $(\mathrm{L})$ and high $(\mathrm{H})$ confinement mode shots, but also important experimental features

\footnotetext{
* Corresponding author: e-mail: apigarov@ferp.ucsd.edu, Phone: +1 8588224916, Fax: +1 8585347716
} 
of a special series of shots focused on edge plasma study, e.g. the so-called Simple-As-Possible-Plasma (SAPP) series using the lowest input power L-mode shots on DIII-D [6,7]. Our results [6] showed the dominance of ACFC in the far SOL transport and the transition to main chamber recycling fueling (MCRF) regime with an increase in the discharge density $\left\langle n_{e}\right\rangle$. An important role of ACFC in plasma transport for H-mode shots was discussed in $[5,6]$.

The detailed description of impurity transport and sources is one of the most challenging issues in the edge plasma physics. Recent experiments carried out on C-Mod (see Ref. [10]) and DIII-D (see Ref. [11]) tokamaks indicated that the main chamber walls could be an important source of impurities. These observations are consistent with fast non-diffusive transport in the SOL. Moreover, recent experiments on non-diffusive transport in DIII-D edge [12] and theoretical models [9, 13] have shown two kinds of intermittent objects: plasma density "blobs" and "holes". The blob objects, which contain a hot and dense plasma, propagate radially toward the chamber wall. In contrast, the hole objects, which carry smaller (near-wall) plasma density, propagate inward. It is expected [9] that these holes play important role in the non-diffusive transport of low impurity charge states by enhancing the radial transport of these ions. The effect of holes on impurity transport should be carefully investigated and incorporated into the edge codes.

As the next step in our studies, we discuss the ACFC model for impurity and main plasma ions. We apply the multi-fluid code analysis on medium size tokamaks: DIII-D, C-Mod, and NSTX. The results of the present paper show that edge plasma in all these tokamaks can be simulated under the same assumptions of anomalous multi-fluid cross-field transport.

\section{Model for 2D profile of anomalous cross-field convective velocity}

In our cross-field transport model, the convective term plays very important specific role. This term provides transport of plasma ions with the velocity $V_{c o n v}\left(\psi_{n}, \theta\right)$ in a given direction. For the majority ion species, this anomalous convective flux is due to averaging over blobs causing infrequent, but large, transport events directed outward. The typical profile of plasma $V_{c o n v}$ is shown in Fig.1. The $V_{c o n v}$ strongly increases with minor radius. The radial gradient of $V_{c o n v}$ is given in the normalized poloidal magnetic flux space $\psi_{n}$. On a given magnetic flux surface, $V_{c o n v}$ has maximum at the outer mid-plane. The poloidal ( $\theta$-coordinate) profile is updated with respect to the profile used previously in [5]. An important finding of recent UEDGE simulations is that significant anomalous convection should occur on the inboard part of the torus. One possible reason is that the blob movement outward on the outboard side is a cause of strong MHD perturbation to the magnetic flux tube that may result in this tube moving toward the wall on the inboard side. The inboard/outboard asymmetry is typically $1: 3$. In the divertor and private flux regions, $V_{c o n v}$ is taken about $1 / 3$ of its mid-plane value. Typically, these values of $V_{\text {conv }}$ in the divertor are used to match a set of diagnostics in this region, for example, the Langmuir probe, IR TV, and Thomson laser scattering (TLS) on DIII-D.

The $V_{\text {conv }}\left(\psi_{n}, \theta, z\right)$ profile for impurity ions is the focus of our present studies. In our UEDGE code model, each impurity ion charge state is assigned its own convective velocity:

$$
V_{\text {conv }}\left(\psi_{n}, \theta, z\right)=V_{\text {conv }}^{B P}\left(\psi_{n}, \theta\right) * \Phi_{z}(z),
$$

where $V_{\text {conv }}^{B P}$ is the ACFC velocity of background plasma (i.e. of $D^{+}$ions), $\Phi_{z}(z)$ is the distribution function (DF), and $z$ denotes the charge state of the impurity ion, $z \geq 1$. The $\Phi_{z}(z)$ distribution suggests that the lowest impurity charges states are either partially or completely entrained in plasma density holes, so that their $V_{\text {conv }}$ is either strongly reduced in comparison with background plasma convective velocity or completely directed toward the core plasma, respectively. At the same time, the highest impurity charge states behave more like the main plasma ions, i.e. these states are entrained into blobs predominantly moving toward the wall. Hereafter, $V_{\text {conv }}\left(D^{+}\right)$and $V_{\text {conv }}\left(C^{+z}\right)$ denote the local values of ACFC velocity for $D^{+}$and $C^{+z}$ ions, respectively. For the DF $\Phi_{z}(z)$ of carbon ions, we use the $\Phi_{C C C}(z)$ distributions which have $V_{\text {conv }}\left(C^{+z}\right)<V_{\text {conv }}\left(C^{+(z-1)}\right)$, $V_{\text {conv }}\left(C^{+6}\right)=V_{\text {conv }}\left(D^{+}\right)$, and $-1 \leq V_{\text {conv }}\left(C^{+z}\right) / V_{\text {conv }}\left(D^{+}\right) \leq 1$ and which are characterized by a single parameter $\eta_{c c c}=\left[V_{\text {conv }}\left(C^{+1}\right)+V_{\text {conv }}\left(C^{+2}\right)+V_{\text {conv }}\left(C^{+3}\right)\right] / V_{\text {conv }}\left(D^{+}\right)$. This parameter continuously decreases from +3 (when all $V_{\text {conv }}$ are the same as for $D^{+}$) down to -3 (for the case when $C^{+1}, C^{+2}, C^{+3}$ ions are convected in direction opposite to the $D^{+}$ion direction but at the same speed). In this work, we test different distribution functions $\Phi_{C C C}(z)$ over charge states (some of these DF are shown in Fig.2) searching for those distributions that give better fits to available experimental data. 


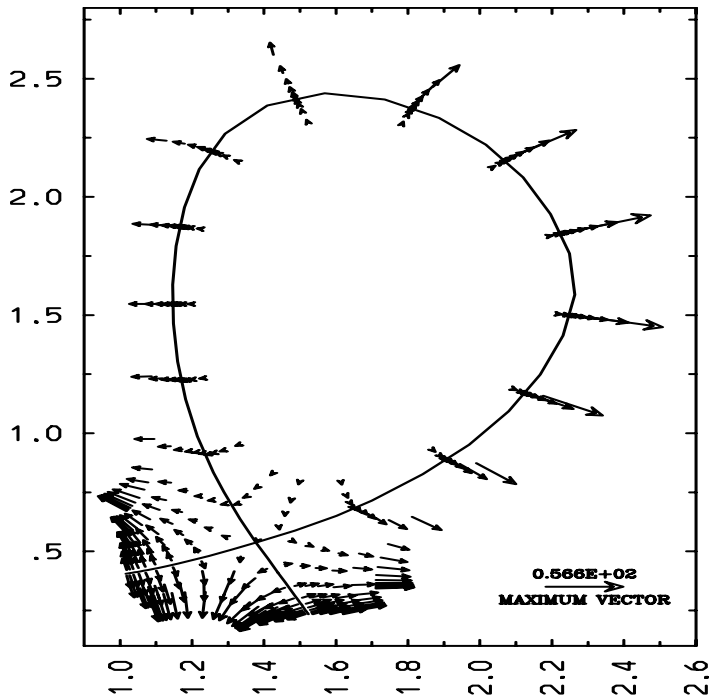

Fig. 1 Vector plot of anomalous convective cross-field velocity $V_{c o n v}$ for majority ion species $\left(D^{+}\right)$related to DIII-D L-mode shot 105500. $\eta_{\text {ece }}=\left(\operatorname{Vconv}\left(C^{+1}\right)+V \operatorname{conv}\left(C^{+2}\right)+V \operatorname{conv}\left(C^{+3}\right)\right) / V \operatorname{conv}\left(D^{+}\right)$

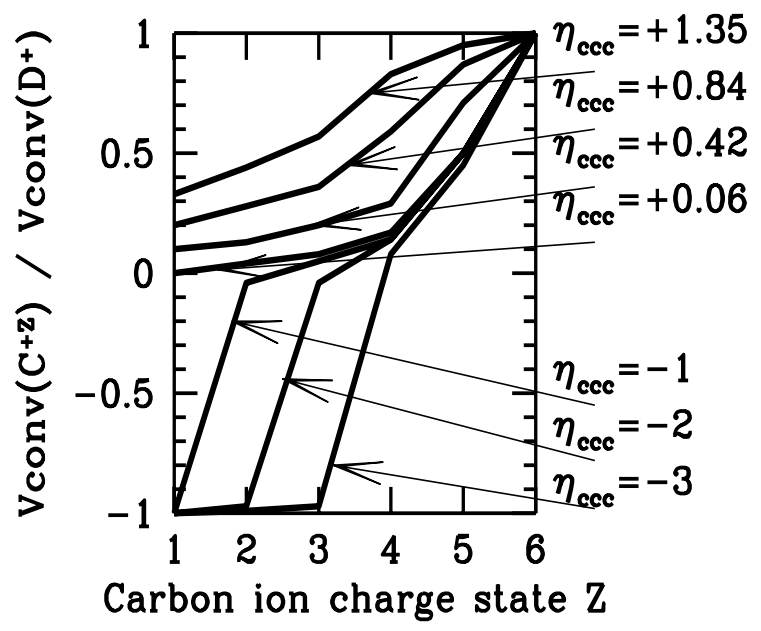

Fig. 2 Model distributions of anomalous convective velocity $V_{c o n v}$ for carbon ion charge states $C^{+z}$. This family of distributions is characterized by parameter $\eta_{c c c}$.

\section{Results of impurity transport modeling with UEDGE}

With the UEDGE code, we simulate multi-fluid transport in a series of L-mode discharges obtained on DIII-D, NSTX, and C-Mod. In DIII-D and NSTX cases, we model carbon impurities (atoms and six charge states), while for C-Mod, we use boron impurities. We use realistic impurity sources due to physical sputtering, chemical sputtering, and self-sputtering (the sputtering coefficients are similar to those incorporated into the DIVIMP code [14]). We assume $D_{\perp}$ and $\chi_{\perp}$ to be constants outside the separatrix. In the core plasma region, these coefficients are taken to decrease slightly (and strongly, for NSTX) in the radial direction ( $\psi_{n}$-space) for a better match to the in/out SOL profile asymmetry.

At present, a simple diffusive model is used in UEDGE to describe the transport of impurity neutrals. The characteristic scale length in diffusive model is $\lambda_{C 0}=\left\{2 T_{C 0} /\left[M_{C} \nu_{i}\left(\nu_{i}+\nu_{c x}+\nu_{e l}\right)\right]\right\}^{1 / 2}$, where $\nu_{i}, \nu_{c x}, \nu_{e l}$ are the frequencies for ionization, charge exchange, and elastic scattering of an atom, $M_{C}$ is the mass of carbon atom. It is assumed that effective neutral gas temperature is $T_{C 0}=\zeta_{t h} T_{i}+\left(1-\zeta_{t h}\right) T_{s p t}$, where parameter $0 \leq \zeta_{t h} \leq 1$ determines the fraction of thermalized atoms, $T_{s p t} \approx 1 \mathrm{eV}$ is the characteristic temperature of primary sputtered particles. This is crude approximation, which introduces an additional uncertainty related to impurity atom penetration. Typically, ionization of carbon atoms is the dominant process in the SOL, so we take $\zeta_{t h}=0.01$ in most of our calculations.

Here we consider an interesting case related to the lowest density SAPP-series shot 105500 on DIII-D. Amongst the SAPP shots, this shot has the highest concentration of $C^{+6}$ impurity measured in the core plasma, whereas the particle flux to chamber walls is the lowest. Shot 105500 has been already modelled with UEDGE in Ref. [6] based on fixed impurity fraction model. This time, we perform a more detailed, multi-fluid analysis of the shot. We focus on the study of the effect of anomalous convection of impurities on edge plasma parameters. The detailed comparison between experimental and UEDGE data for the hydrogen and carbon ion spectroscopy for this shot is given in [15] (including the realistic 3D model of visible light reflection from the chamber wall).

In our analysis we use the following three basic parameters: (i) the peak temperature at the outer divertor plate ( $T_{e d}$ ), (ii) the relative concentration $\eta_{C 6}=\left[C^{+6}\right] / n_{e}$ of $C^{+6}$ ions at the mid-plane on the $\psi_{n}=0.95$ magnetic flux surface, and (iii) the mid-plane horizontal view chord brightness $B_{\text {mid }}(C I I I)$ of $C$ III-ion line at $4650 \AA$. These three parameters are calculated by UEDGE and routinely measured on DIII-D, respectively, with Langmuir probes and divertor TLS system, with neutral-beam charge exchange recombination spectroscopy (NB CERS) in combination with TLS system for the SOL profiles of plasma density and temperature, and with fast filterscopes. The concentration of fully ionized carbon $\left(C^{+6}\right), \eta_{C 6}$, depends strongly on both edge transport and sputtering 
sources. The $B_{\text {mid }}(C I I I)$ brightness may characterize the carbon ion sources in the main chamber, whereas $T_{e d}$ is indicative of plasma state in the divertor.

First of all, we answer the following question: can anomalous cross-field transport of impurity ions be purely diffusive in the case when background plasma transport is dominated by the radial intermittent convection? We perform two series of UEDGE runs and present our results in Fig.3. In the first series (curves with filled hexagons), we increase the diffusion coefficient $D_{\perp}(z)$ for all impurity ions by the same factor with respect to $D_{\perp}\left(D^{+}\right)$of background plasma. As seen, the simple increase in impurity species diffusion only increases the inconsistency between calculated and experimental data. For instance, an increase in $D_{\perp}$ results in $\eta_{C 6}$ decreasing since the leakage of $C^{+6}$ and $C^{+5}$ ions from the core region increases more strongly than the fluxes of lower charge state ions into the core. In the second series (curves with open circles), we assume the following distribution function for local impurity diffusion coefficient: $D_{\perp}\left(C^{+}\right)=D_{\perp}\left(D^{+}\right) \gamma_{C I}$ for $C^{+}$-ion and $D_{\perp}(z)=D_{\perp}\left(D^{+}\right)\left[1+\left(\gamma_{C I}-1\right) / z^{2}\right]$ for other $C^{+z}$ carbon ions, where parameter $\gamma_{C I} \geq 1$. In this series, experimental levels of $T_{e d}$ and $\eta_{C 6}$ can be reached only at very high values of $D_{\perp}\left(C^{+}\right) / D_{\perp}\left(D^{+}\right)$but calculated $B_{\text {mid }}$ is higher than that measured. Moreover, the fact that the required values of $D_{\perp}\left(C^{+}\right)$here exceed the Bohm value by more than a factor of 10 is indicative of non-diffusive transport of impurities in the SOL. So, purely diffusive models are unsuitable for description of impurity transport when anomalous convection dominates in the SOL plasma transport.

Next, we run UEDGE with different ACFC velocity distribution functions $\Phi_{C C C}(z)$ over charge states $z$ of carbon impurity ion (Fig.2). For each test distribution we adjust $V_{c o n v}^{B P}, D_{\perp}, \chi_{\perp}$, and wall albedo to match the radial SOL plasma density and temperature profiles, core particle balance, $D_{\alpha}$ signals measured by horizontal and tangential filterscopes at mid-plane, and mid-plane gas pressure. The diffusivity coefficients, $D_{\perp}$ and $\chi_{i \perp}$, are taken to be the same for all ion species. In doing so, we found (as in Refs. [5, 6]) that anomalous plasma convection is the dominant plasma transport process in the SOL.

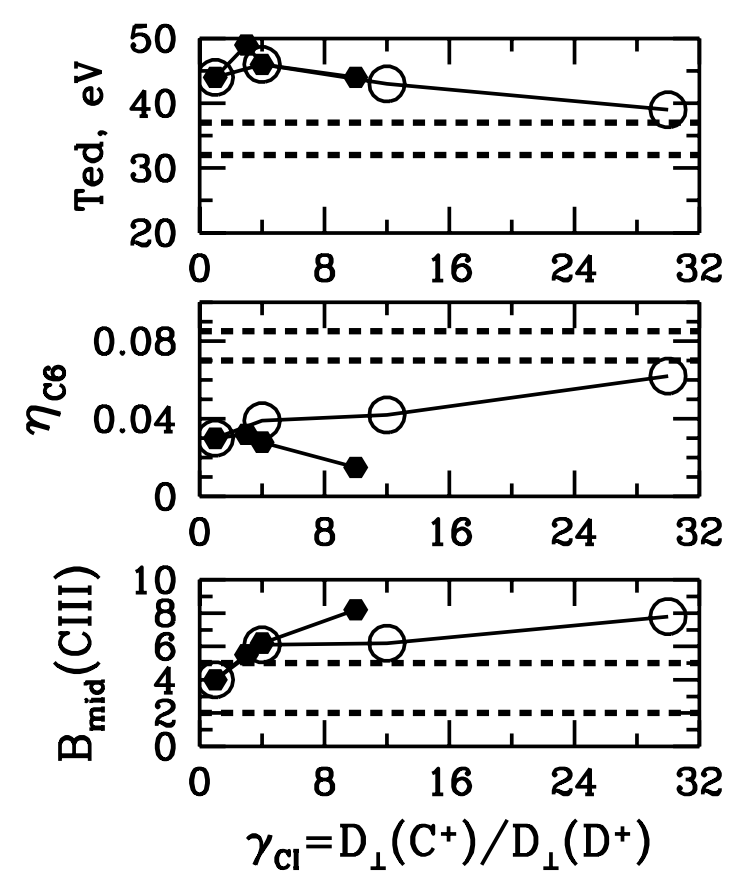

Fig. 3 Variation of $T_{e d}, \eta_{C 6}$, and $B_{m i d}(C I I I)$ with the ratio $\gamma_{\mathrm{CI}}$ of cross-field diffusion coefficients of carbon and deuterium ions $\left(D_{\perp}\left(C^{+}\right)\right.$and $\left.D_{\perp}\left(D^{+}\right)\right)$On each panel, experimental DIII-D data are scattered between two horizontal broken lines.
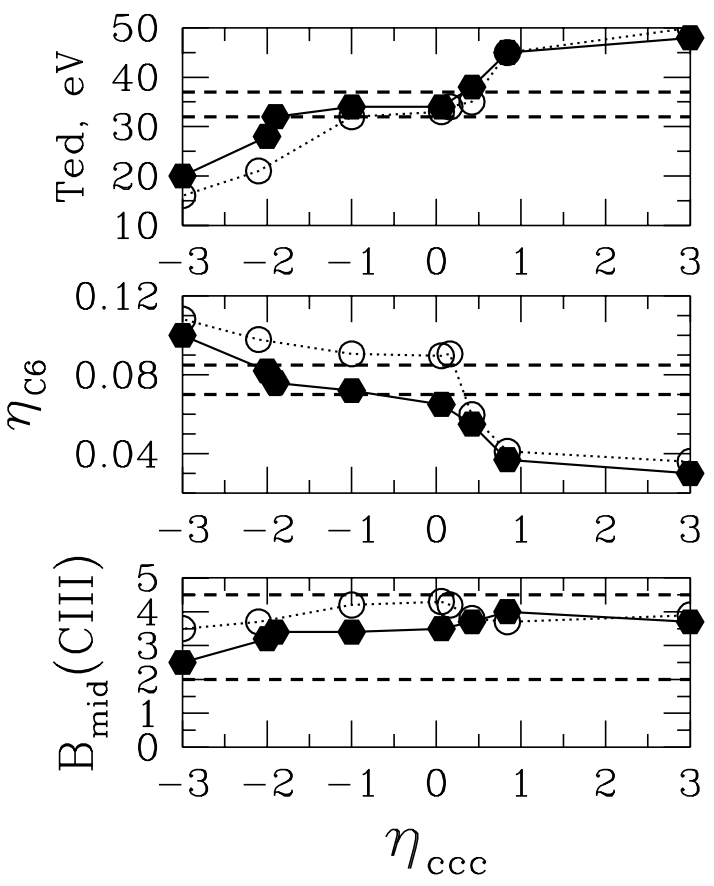

Fig. 4 Variation of $T_{e d}, \eta_{C 6}$, and $B_{\text {mid }}(C I I I)$ with impurity model parameter $\eta_{c c c}$ calculated for DIII-D shot 105500 by using $\zeta_{t h}=0.01$ (solid curves) and $\zeta_{t h}=1$ (dotted curves). On each panel, experimental DIII-D data are scattered between two horizontal broken lines.

The results of UEDGE simulations including anomalous impurity ion convection are given in Figs. 4,5 for the DIII-D shot under consideration. In Fig.4, we compare the results obtained for low ( $\zeta_{t h}=0.01$, solid lines 
with hexagons) and high ( $\zeta_{t h}=1$, dotted lines with circles) penetration of carbon atoms. In both cases, $T_{e d}$ and $\eta_{C 6}$ are strong functions of $\eta_{c c c}$. The weaker the transport of atoms through the SOL, the stronger should be the inward cross-field convective transport of few lowest charge states of carbon ion in order to match the measured level of core plasma contamination $\eta_{C 6}$. As seen, if $\eta_{c c c} \geq 0.5$, i.e. $V_{c o n v}$ for all impurity ions is directed toward the wall, most of the experimental data is not matched with UEDGE. In the realistic case when $\zeta_{t h}$ is small, experimental values are well matched with UEDGE in a wide range $-2.5 \leq \eta_{c c c} \leq-1$ when $C^{+}$and $C^{++}$ions are rapidly convected inward. The chemical sputtering (CS) yield is very uncertain at surface temperatures close to room temperature and low energies of incident particles that is typical for L-mode. The effect of CS yield uncertainty is studied in Fig.5. We display UEDGE results without (curves with open circles) and with (curves with filled hexagons) chemical sputtering of the wall at $T_{w}=300 \mathrm{~K}$ for $\zeta_{t h}=0.01$. As seen, the smaller the sputtering source, the more actively the lowest charge states should be convected inward in order to match experimental data.

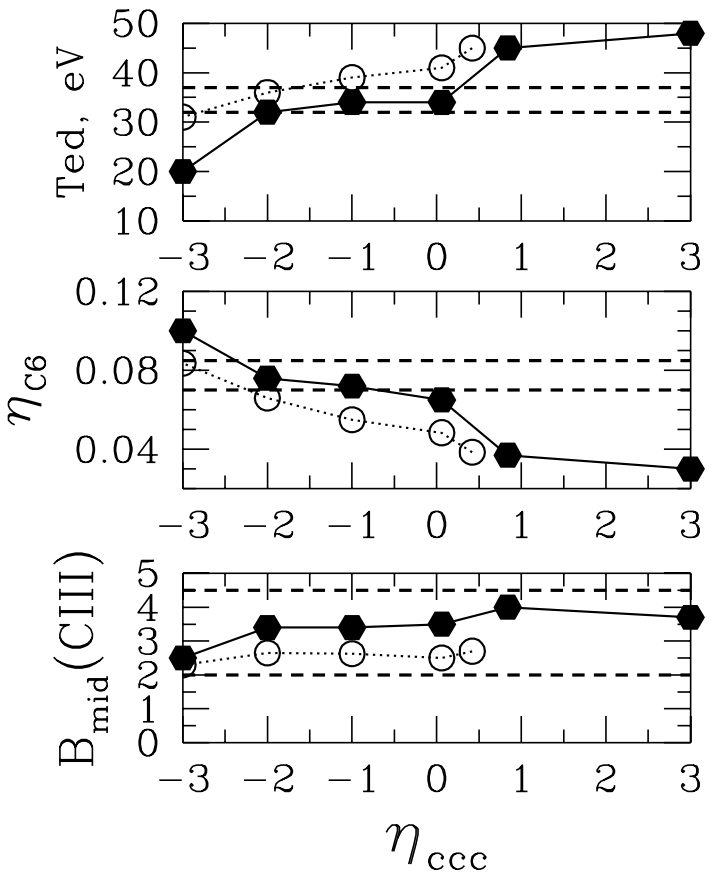

Fig. 5 Variation of $T_{e d}, \eta_{C 6}$, and $B_{\text {mid }}(C$ III) with impurity model parameter $\eta_{c c c}$ calculated for DIII-D shot 105500 with (solid curves) and without (dotted curves) chemical sputtering of the chamber wall.
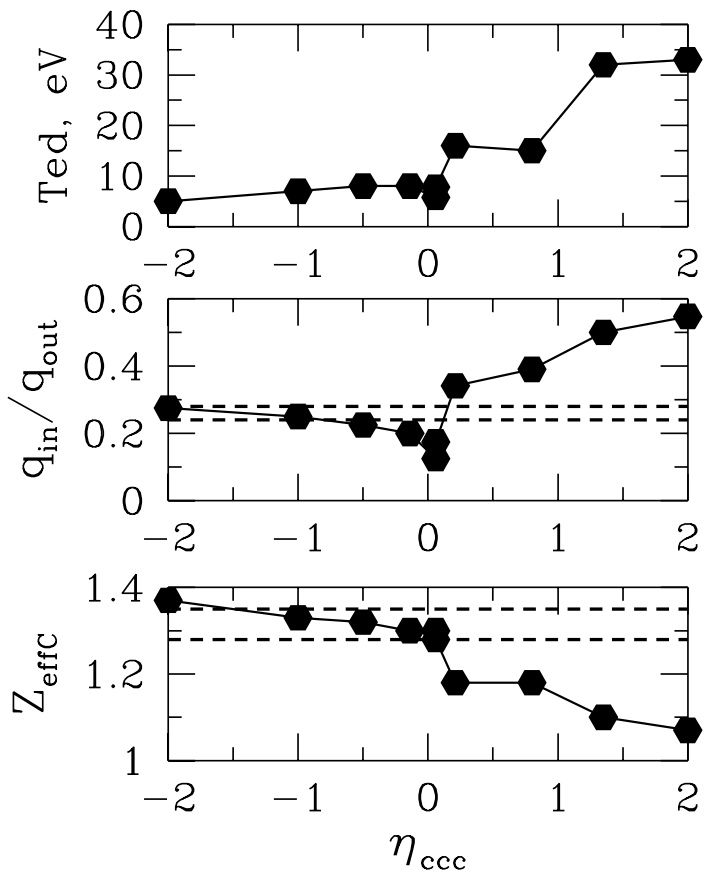

Fig. 6 Variation of $Z_{e f f C}$, inner plate peak $T_{e d}$, and $q_{\text {in }} / q_{\text {out }}$-ratio for NSTX shot 109033 . Experimental NSTX data are scattered between two horizontal broken lines.

Important results were also obtained in the multi-fluid modelling of edge plasma for NSTX spherical tokamak. We simulate the low-power L-mode shot 109033 at $253 \mathrm{~ms}$. This is the NSTX shot in which the discharge density $\left\langle n_{e}\right\rangle \approx 2.4 \times 10^{19} \mathrm{~m}^{-3}$ is close to the density of DIII-D shot 105500 considered above. With the UEDGE code, we iteratively match the following: (i) particle flux balance at the core interface consistent with the NBI fueling rate and with the rate of core plasma density increase, (ii) radial profiles of $n_{e}$ and $T_{e}$ measured at mid-plane by TLS on both the inboard and outboard parts of the torus, (iii) $T_{i}$ profile measured by CERS, and (iv) neutral gas pressure measured at outboard mid-plane location. In addition, UEDGE data obtained for different $\eta_{c c c}$ are compared in Fig.6 to the experimental data, namely, the carbon ion $Z_{\text {effC }}$ measured by CERS in the outboard core region around $\psi_{n}=0.7$ and the ratio $q_{\text {in }} / q_{\text {out }}$ measured by IR TV, where $q_{\text {in }}$ and $q_{\text {out }}$ are the peak heat fluxes on the inner and outer divertor plates. As seen on Fig.6 the experimental and simulated data are in good agreement for $\eta_{c c c}<-0.5$, i.e. in the case when non-diffusive impurity transport is strong and the lowest states may be actively convected inward.

The dominant plasma impurity species in recent Alcator C-Mod experiments is boron [11]. In UEDGE modelling, the ACFC velocity profile, $V_{\text {conv }}\left(\psi_{n}, \theta, z\right)$, for boron ions is given by Eq.1. For the distribution function 
$\Phi_{z}(z)$ over charge state of boron ions, $1 \leq z \leq 5$, we use the $\Phi_{B B B}(z)$ distributions which have $V_{\text {conv }}\left(B^{+z}\right)<$ $V_{\text {conv }}\left(B^{+(z-1)}\right), V_{\text {conv }}\left(B^{+5}\right)=V_{\text {conv }}\left(D^{+}\right)$, and $-1 \leq V_{\text {conv }}\left(B^{+z}\right) / V_{\text {conv }}\left(D^{+}\right) \leq 1$ and which are characterized by a single parameter $\eta_{B B B}=\left[V_{\text {conv }}\left(B^{+1}\right)+V_{\text {conv }}\left(B^{+2}\right)+V_{\text {conv }}\left(B^{+3}\right)\right] / V_{\text {conv }}\left(D^{+}\right)$. A family of $\Phi_{B B B}(z)$ distribution functions is shown on Fig.7.

We perform the UEDGE code simulation of impurity transport based on anomalous cross-field convection model for the C-Mod Ohmic heating shot numbered 1030630019. The resulting UEDGE solutions for this shot match the following: (i) particle flux balance at the core plasma interface, (ii) outer SOL profiles of $n_{e}$ and $T_{e}$ measured by TLS and recirprocating probes; (iii) gas pressure in the main chamber at the mid-plane, (iv) $D_{\alpha}$ brightness of selected view sights measured by CCD cameras, and (v) $Z_{\text {eff. }}$. Impurity concentration level in the core plasma is characterized by the effective plasma charge $Z_{e f f}$, the radial profile of which is measured on C-Mod. The variation of calculated $Z_{\text {eff }}$ with parameter $\eta_{B B B}$ in impurity transport model is shown on Fig.8. As seen, the negative values $\eta_{B B B}<-1$ are consistent with the measured level of $Z_{\text {eff }}$ in the core plasma, so that non-diffusive impurity transport may play an important role in core plasma contamination in C-Mod.

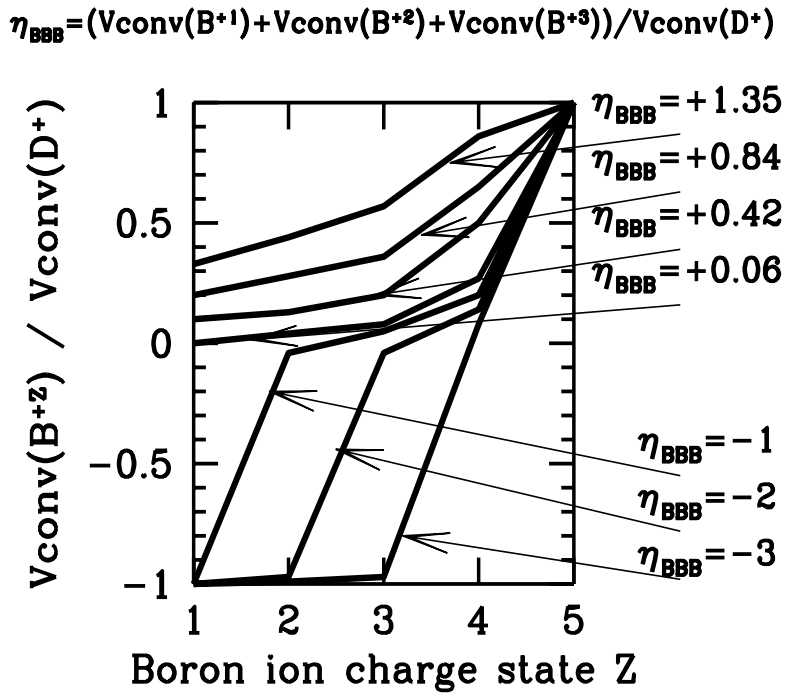

Fig. 7 Model distributions of anomalous convective velocity for boron ion charge states $B^{+z}$ for a set of $\eta_{B B B}$ values.

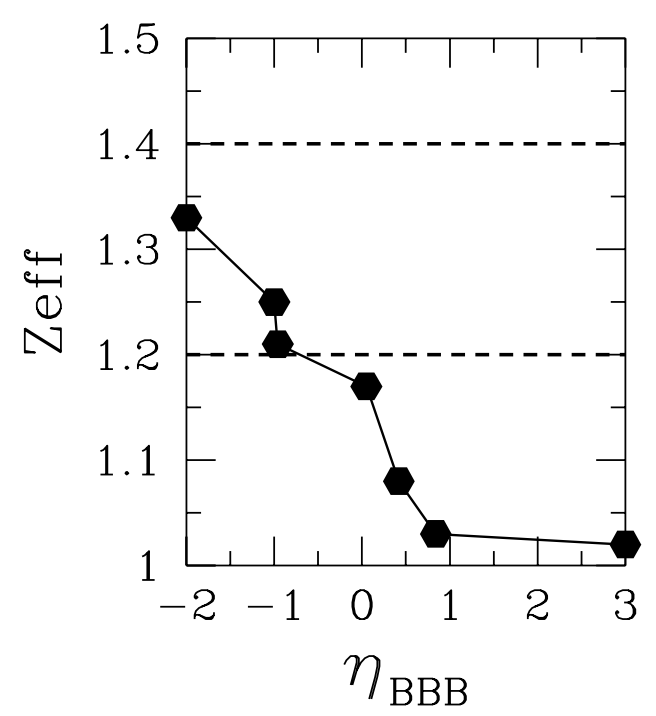

Fig. 8 Variation of $Z_{\text {eff }}$ with impurity model parameter $\eta_{B B B}$ for C-Mod shot 1030630019.

Our recent results of UEDGE modelling for DIII-D, NSTX, and C-Mod suggest that in L-mode shots the chamber wall is important source of impurities and that this source can cause significant contamination of the core plasma. In the case of DIII-D shot, an important role of wall sputtering source can be understood by inspecting Fig.5. The calculated $B_{\text {mid }}$ has a relatively weak dependence on $\eta_{c c c}$ since impurity sputtering source is practically not changed while $\eta_{c c c} \geq-2$. Moreover, as follows from the UEDGE calculation with accounting for wall chemical sputtering (WCS), the WCS is 40-50\% of the total sputtering source of wall. As seen, elimination of WCS from the UEDGE modelling results in a reduction in the core impurity concentration $\eta_{C 6}$ by approximately the same $40 \%$ for these plasma conditions.

\section{Some results on cross-field transport comparison in medium size tokamaks}

Using multi-fluid UEDGE to evolve impurities, we have re-done simulations for L-mode SAPP series on DIII-D and confirmed our previous results and major conclusions [6] obtained with a fixed impurity fraction model. In recent modeling we took more uniform poloidal distribution of $V_{\text {conv }}(\theta)$ including the inboard region that allowed us to match the data measured by tangentially as well as horizontally viewing mid-plane filterscopes (see [15]). Solutions were obtained using the same values of $D_{\perp}=D_{\perp}^{\star}=0.15 \mathrm{~m}^{2} / \mathrm{s}$ and $\chi_{\perp}=\chi_{\perp}^{\star}=0.8 \mathrm{~m}^{2} / \mathrm{s}$ for all SAPP shots in a wide range of discharge densities $\left\langle n_{e}\right\rangle$ at the same power input. In addition, we simulated selected Lmode shots of NSTX and C-Mod. Special experimental features (inboard gas puff and inboard/outboard profile asymmetry on NSTX or vertical divertor plates on C-Mod) were modeled. 
We use the simulated SAPP DIII-D data as a baseline for cross-machine comparison. We find that fitted diffusivity coefficients on NSTX and C-Mod tokamaks correspond to the $D_{\perp}^{\star}$ and $\chi_{\perp}^{\star}$ (obtained for DIII-D) being scaled similar to Bohm, $\propto T_{e}^{s} / B_{t}^{s}$, where $T_{e}^{s}$ and $B_{t}^{s}$ are the electron temperature and the toroidal magnetic field at the outer mid-plane on the plasma core boundary surface. The convective velocity at the wall, $V_{c o n v}(w)$, is deduced by matching experimental data on main-chamber recycling, in particular, the mid-plane $D_{\alpha}$ brightness and gas pressure that are $\alpha\left\langle n_{e}\right\rangle^{3}$ for a series of shots (see [6, 9]), yielding a density dependence with $V_{\text {conv }}(w) \propto\left\langle n_{e}\right\rangle^{\beta}, 1 \leq \beta \leq 2$. A nearly linear increase of $V_{c o n v}(w)$ with $\left\langle n_{e}\right\rangle$ is predicted by UEDGE for the SAPP series (DIII-D) and 100517 series (C-Mod) of L-mode shots. Since blobby transport is related to $\vec{E} \times \vec{B}$ drift from polarization, $V_{\text {conv }}$ should decrease inversely with $B_{t}$. For discharges with similar plasma density $\left\langle n_{e}\right\rangle \approx 2.4 \times 10^{19} \mathrm{~m}^{-3}$, we obtain $V_{\text {conv }}(s) \approx 40 \mathrm{~m} / \mathrm{s}$ at the separatrix of low- $B_{t}$ tokamak $\left(B_{t} \approx 0.4 \mathrm{~T}\right.$, NSTX shot $109033)$ vs. $V_{\text {conv }}(s) \approx 10 \mathrm{~m} / \mathrm{s}\left(B_{t} \approx 1.9 \mathrm{~T}\right.$, DIII-D shot 105500$)$. For C-Mod $\left(B_{t} \approx 5.6 \mathrm{~T},\left\langle n_{e}\right\rangle \approx 10^{20} \mathrm{~m}^{-3}\right.$, shot $100517017)$, we obtained $V_{\text {conv }}(s) \approx 10 \mathrm{~m} / \mathrm{s}$, i.e., $V_{\text {conv }}$ may scale as a combination of $\left\langle n_{e}\right\rangle$ and $B_{t}$. For shots considered, the best agreement between simulated and experimental data gives the impurity convection parameter as $-2 \leq \eta_{c c c} \leq-0.5$. A detailed comparison of cross-field transport in tokamaks will be reported elsewhere.

\section{Conclusions}

We have used the multi-fluid transport code UEDGE to perform extensive simulations for L-mode edge plasmas in medium size tokamaks including C-Mod, DIII-D, and NSTX. Our previous and recent results show that fast non-diffusive intermittent plasma transport (which is modeled by UEDGE as anomalous cross-field convection) can be the dominant SOL transport mechanism in all these tokamaks. By matching the main chamber recycling data in tokamaks, we find that anomalous convective velocity in the far SOL should increase with discharge density $\left\langle n_{e}\right\rangle$. Moreover, cross-tokamak comparison shows that anomalous convective velocity should increase with decrease in toroidal field strength in agreement with theoretical models and measurements of edge turbulence indicating that non-diffusive transport is related to $\vec{E} \times \vec{B}$ drift from polarization.

In our modeling of different tokamaks, the best agreement between the calculated and experimental data gives the impurity cross-field transport model in which anomalous cross-field convection dominates in the SOL transport, the background plasma ions and high charge state impurity ions are convected toward the wall, while the low charge state impurity ions either are convected toward the core plasma or, at least, their convection outward together with majority ion species is significantly reduced. Our analysis also shows that impurities from wall can cause significant contamination of core plasma if impurity transport in the SOL is fast and non-diffusive. Therefore, for further validation of impurity model, we intend to expand our analysis on matching the multi-fluid code results with an extensive set of DIII-D experimental spectroscopic data for each impurity ion charge state and more detailed data from impurity transport experiments on other tokamaks.

Acknowledgements We are grateful to Dr. R. Bell (PPPL) for making NB CERS measured data for NSTX shot \#109033 available to us. The work was performed under the auspices of the U.S. Department of Energy by UCSD under grant No. DEFG03-00ER54568 and by University California LLNL under contract No. W-7405-Eng-48.

\section{References}

[1] B. LaBombard et al., Phys. Plasmas 8, 2107 (2001); B. Lipschultz et al., Plasma Phys. Control. Fusion. 44, 733 (2002).

[2] J.A. Boedo et al., Phys. Plasmas 8, 4826 (2001); 10, 1670 (2003).

[3] J.A. Boedo,"Edge reciprocating probe results", NSTX Research Forum, September 2002, http://nstx.pppl.gov.

[4] S.I. Krasheninnikov, Phys. Lett. A 283, 368 (2001).

[5] A.Yu. Pigarov et al., Phys. Plasmas 9, 1287 (2002).

[6] A.Yu. Pigarov et al., J. Nucl. Mater. 313-316, 1076 (2003).

[7] P.C. Stangeby et al. J. Nucl. Mater. 313-316, 883 (2003).

[8] T.D. Rognlien et al., J. Nucl. Mater. 196, 347 (1992).

[9] S.I. Krasheninnikov, A.Yu. Pigarov, et al., online Proceedings of the 19th IAEA Conf., CN-94/TH/4-1 (2002).

[10] W. P. West et al., J. Nucl. Mater. 313-316, 1211 (2003).

[11] B. Lipschultz et al., "Comparison of low-z and high-z impurity influxes in Alcator C-Mod", PSI Conference presentation, Toki, Japan (2002), http://www.psfc.mit.edu/people/blip/

[12] J. A. Boedo et al., J. Nucl. Mater. 313-316, 813 (2003).

[13] G.Q. Yu, S.I. Krasheninnikov, Phys. Plasmas 10, 4413 (2003); also "2D modelling of blob dynamics", this conference.

[14] P.C. Stangeby, J. Elder, J. Nucl. Mater. 220-222, 193 (1995).

[15] E. Hollmann, A. Pigarov, "Multi-fluid code simulation of DIII-D filterscope data including reflection...", this conference. 